\title{
Preparación de los estudiantes de Cultura Física para la atención a la diversidad educativa desde la Práctica Laboral Investigativa
}

\author{
Preparation of the students of Physical Culture for the attention to the \\ educational diversity from the Investigative Labor Practice \\ Preparação dos alunos da Cultura Física para a atenção à diversidade \\ educacional da Prática Investigativa do Trabalho
}

IDALIA CLEJEL TOIRAC ${ }^{1}$

\begin{abstract}
RESUMEN
Esta investigación analiza las insuficiencias en la preparación de los estudiantes para atender la diversidad educativa durante la Práctica Laboral Investigativa y en especial, en la Educación Física, limitando su desempeño preprofesional. Para ello utiliza los métodos teóricos y empíricos propios de la investigación pedagógica. El trabajo aporta un modelo pedagógico, asumiendo la preparación del estudiante de tercer año para atender la diversidad educativa como una cualidad superior en su modo de actuación preprofesional. Contribuye a un mejor desempeño preprofesional diversificado desde la Práctica Laboral Investigativa en la Educación Física. Los resultados generales se concretan en un movimiento ascendente en los indicadores establecidos para evaluar el nivel de preparación de los estudiantes. Tanto el modelo pedagógico como la metodología fueron avalados por especialistas cubanos.
\end{abstract}

Palabras clave: Formación inicial; preparación; atención a la diversidad educativa; practica laboral investigativa.

\begin{abstract}
This research analyzes the insufficiencies in the students' preparation to attend the educational diversity during the Investigative Labor Practice and especially, in the Physical Education, limiting their pre-professional performance. For this, it uses the theoretical and empirical methods of pedagogical research. The work provides a pedagogical model, assuming the preparation of the third year student to attend educational diversity as a higher quality in their pre-professional mode of action. It contributes to a better diversified pre-professional performance from the Investigative Labor Practice in Physical Education. The general results are reflected in an upward movement in the established indicators to assess the level of students' preparation. Cuban specialists endorsed both, the pedagogical model and the methodology.
\end{abstract}

Keywords: Initial training; preparation; attention to educational diversity; investigative work practice.

1. UNIVERSIDAD DE ORIENTE, Cuba | iclejel@uo.edu.cu 


\section{RESUMO}

Esta pesquisa analisa as insuficiências na preparação dos alunos para atender à diversidade educacional durante a Prática Investigativa do Trabalho e, principalmente, na Educação Física, limitando seu desempenho pré-profissional. Para isso, utiliza os métodos teóricos e empíricos da pesquisa pedagógica. $\mathrm{O}$ trabalho fornece um modelo pedagógico, assumindo a preparação do aluno do terceiro ano para atender à diversidade educacional como uma qualidade superior em seu modo de ação pré-profissional. Contribui para um desempenho pré-profissional mais diversificado da Prática Laboral Investigativa em Educação Física. Os resultados gerais são refletidos em um movimento ascendente nos indicadores estabelecidos para avaliar o nível de preparação dos alunos. Ambos, o modelo pedagógico e a metodologia foram endossados por especialistas cubanos.

Palavras-chave: Treinamento inicial; preparação; atenção à diversidade educacional; prática de trabalho investigativo.

\section{INTRODUCCIÓN}

La formación como categoría es estudiada por la Pedagogía y la Psicología, entre otras ciencias como un proceso social que reconoce el carácter consciente del desarrollo humano y su naturaleza holística, compleja y dialéctica. Autores como Vaillant (2001, p. 34); Moreno (2003, p. 20); Calzado (2004, p. 10); Díaz (2005, p. 8) y otros han realizado valiosas consideraciones al respecto. En general, valoran la formación inicial como un proceso, una función o una capacidad evolutiva, como una actividad que tiene como máxima pretensión el desarrollo de las potencialidades del individuo.

Asimismo, Vinent (2000, p. 16); Bermúdez (2002, p. 6); Venet (2007, p. 5) \& Suárez (2003, p. 8) han estudiado la formación en estudiantes del nivel superior, preuniversitario y escolares de la educación primaria, respectivamente. Son coincidentes sus puntos de vistas, destacando que el proceso formativo prepara a los estudiantes para dirigir grupos de sujetos de diferentes particularidades individuales. El tema de la atención a la diversidad desde la formación inicial constituye también un núcleo de interés para los sistemas educativos de las universidades cubanas debido al carácter novedoso, polisémico, multireferencial que representa en la actualidad para la ciencia y el encargo social de educar para la vida en una sociedad pluricultural cada vez más numerosa y diversa.

De manera general, la atención a la diversidad es abordada por autores como Guerra (2005, p. 78); Aldea (2006, p. 35); Venet (2007, p. 8); Prada (2014, p. 78); Blanco (2015, p. 29); Marchesi (2015, p. 95); Jaur (2017, p. 267), entre otros que han dirigido sus estudios hacia la búsqueda de referencias en el contexto nacional y mundial, revelando las particularidades esenciales de la formación competitiva profesional en la diversidad de los futuros pedagogos en función del desarrollo de la personalidad y en virtud de dicha estimulación.

Igualmente se ha analizado desde la formación inicial, la Práctica Laboral Investigativa (en lo adelante PLI) para las carreras de corte pedagógico, abordada por investigadores como 
Addine (1996, p. 13) \& García (2004, p. 10) desde su relación con los componentes laboral e investigativo. Castillo (2002, p. 11), con el trabajo metodológico de su colectivo. Y Velázquez (2003, p. 12) relacionado con la formación de profesores de Secundaria Básica para conducir el desarrollo de las habilidades profesionales. Reconociendo los aportes de Castillejo (2004, p. 10) desde la superación en la Educación Física durante el adiestramiento laboral, Milán $(2005$, p. 33) como proceso pedagógico profesional permanente en el entrenador deportivo, Novoa (2006, p. 10) desde sus contenidos en torno a la función cultural de la familia, entre otros autores.

En Cuba, la carrera de Cultura Física, inmersa en un continuo proceso de perfeccionamiento, ha provocado interesantes modificaciones en la formación inicial de sus profesionales. En este sentido, se destacan las indicaciones del Ministerio de Educación Superior referidas a la modelación del perfil amplio de este egresado contentivo de cuatro esferas de actuación profesional como Licenciado en Cultura Física: Educación Física, Recreación Física, Cultura Física Profiláctica y Terapéutica y el Deporte. Esta particularidad en el modelo de formación inicial de la carrera Cultura Física convierte en ineludible la necesidad de que el estudiante durante su paso por los diferentes años académicos y su interacción con las esferas de actuación profesional a través de la PLI, se apropie de las herramientas y recursos psicopedagógicos indispensables para atender la diversidad, dada la complejidad de cada contexto, persona o grupo donde se desempeñe.

Existen trabajos científicos en el marco de la formación inicial del estudiante de la Cultura Física, pero en un plano general Hechavarría $(2006$, p. 10) \& Oroceno $(2008$, p. 9) se proyectan directamente a la formación del entrenador deportivo, particularizando en su entrenamiento. Mendoza (2007, p. 12) aporta la necesidad de favorecer la atención a la diversidad desde el trabajo interdisciplinario. Rabilero (2010, p. 11); Robert (2009, p. 9) \& Ramírez (2009, p. 9) han realizado aportes para dirigir, orientar el crecimiento personal y profesional de los estudiantes en la formación inicial. Castillo (2014, p. 11) la superación profesional desde habilidades relacionadas con el trabajo comunitario y Mataran (2015, p. 65 ) el protagonismo del estudiante universitario en el proceso de enseñanza-aprendizaje.

De manera general, estas investigaciones son valiosas pues se reconocen las variadas aportaciones en materia de atención a la diversidad; sin embargo no aportan suficientes argumentos para afrontar desde la formación inicial del estudiante de Cultura Física las limitantes que obstaculizan el desempeño preprofesional diversificado en los egresados, además no profundizan en cómo llevar a cabo, los métodos y procedimientos para conducir este proceso; sobre todo teniendo en cuenta el dominio de hábitos, habilidades y procederes que les permita aprovechar las potencialidades físicos, psicomotrices, psíquicas, educativas y sociales en los diversos sujetos con los que debe trabajar.

En consecuencia, con el análisis anterior se valora como demanda científica la necesidad de una lógica formativa cuya dinámica epistemológica garantice durante la formación inicial del Licenciado en Cultura Física, la apropiación de los recursos teóricos y metodológicos imprescindibles para un desempeño preprofesional diversificado a partir de los diferentes contextos de actuación que enfrente el egresado. 


\section{METODOLOGÍA}

Se seleccionó una población conformada por 114 estudiantes de segundo año del curso diurno en la facultad de Cultura Física en Santiago de Cuba, curso escolar 2016-2017. La muestra estuvo integrada 38 por 58 estudiantes para un $50,8 \%$ de la población seleccionada. Se trabajó además con 18 profesores del colectivo pedagógico que responden al segundo año de la carrera, entre los que figuran los profesores guías y el profesor principal del año académico. El criterio de selección de la muestra es intencional, siendo interés de la investigación aquellos estudiantes que desarrollan su PLI en escuelas primarias del municipio Santiago de Cuba de manera particular en el segundo ciclo (quinto y sexto grado). Justamente, son los estudiantes que trabajan en dicho ciclo los que presentan mayores dificultades en la atención a la diversidad de los escolares primarios y por consiguiente donde se presentan las mayores dificultades en la PLI.

Para dar cumplimiento al objetivo se utilizaron métodos teóricos, empíricos y técnicas de carácter empírico como la observación a actividades docentes y extradocentes, entrevistas a los profesores universitarios, maestros primarios, tutor y la encuesta a estudiantes. Para la realización del diagnóstico se estableció un sistema de indicadores de diagnóstico que permitió orientar científicamente su desarrollo. Indicadores del diagnóstico: 1. Formación psicopedagógica a los estudiantes para la PLI de Educación Física. 2. Preparación psicopedagógica de los estudiantes durante la PLI de Educación Física. 3. Atención a la diversidad por parte de los estudiantes durante la PLI de Educación Física.

\section{RESULTADOS}

\section{Estructura funcional de la preparación de los estudiantes de Cultura Física para la atención a la diversidad educativa durante la PLI}

La preparación del estudiante de Cultura Física para la PLI orientada a la atención a la diversidad educativa, es un proceso que tiene como partida, su concepción y planificación, en el que los problemas profesionales, objetivo, contenido, métodos, así como formas de organización y evaluación; constituyen el principal recurso preparatorio que posibilita las herramientas pedagógicas al estudiante para operar con las respuestas educativas diversificadas y ajustadas a las necesidades educativas de los estudiantes, desde sus características físico-motoras hasta la dinámica de las relaciones interpersonales del grupo y la personalidad, para facilitar el logro de los objetivos educativos atendiendo al grupo etario de desarrollo.

Partiendo de los criterios concertados anteriormente, se conciben las dimensiones en la preparación del estudiante de licenciatura en Cultura Física para la atención a la diversidad educativa: la dimensión orientadora contextualizada de la PLI, la dimensión aplicativa de la PLI y la dimensión valorativa de la PLI que desde una interpretación holística contribuyen al dominio de las habilidades de la profesión, sobre la base de la puesta en práctica de la relación dialéctica entre lo objetivo y lo subjetivo para dar respuestas pedagógicas apropiadas a las situaciones profesionales, es decir a los acontecimientos relacionados con el contenido de la profesión que surgen y suceden en los procesos que tienen lugar en los diferentes ámbitos de desempeño profesional y social en general. 
Para revelar la dinámica desde la dimensión orientadora contextualizada de la PLI, se establecen las configuraciones: organizativa Práctico Laboral Investigativa que es el proceso que media con la configuración didáctica Práctico Laboral Investigativa.

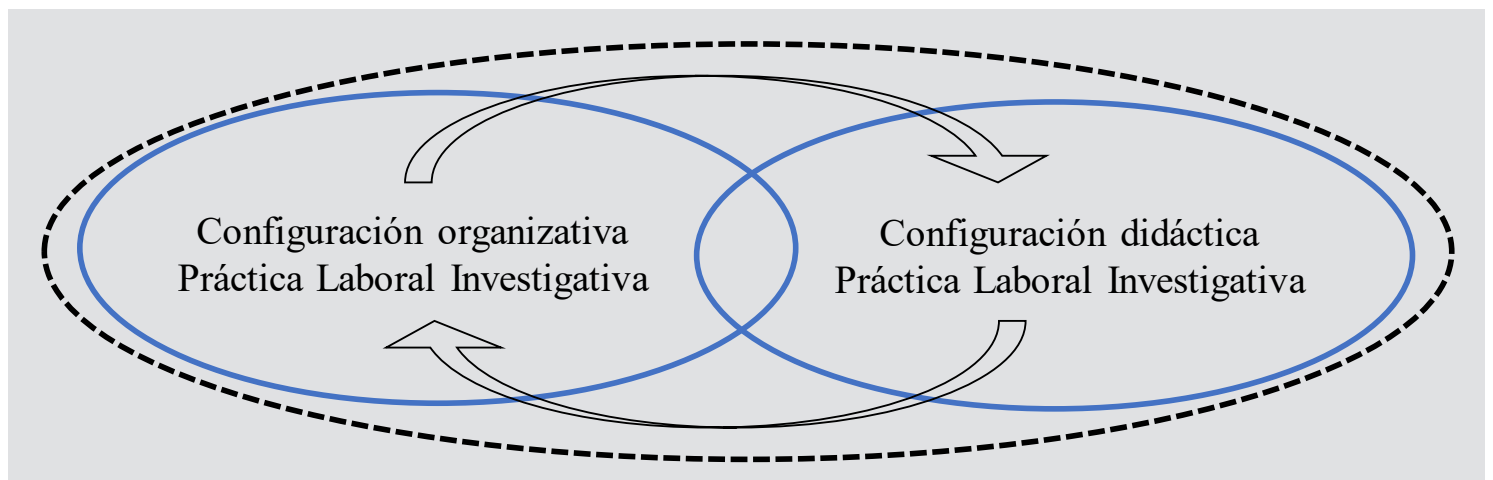

Figura 1. Esquema de la dimensión orientadora contextualizada de la PLI

Desde esta óptica esta primera dimensión orientadora contextualizada de la PLI, sugiere el establecimiento de las coordenadas pedagógicas necesarias para enfrentar la diversidad de situaciones en la que se presentan sus manifestaciones en la comunidad educativa, en lo físico motor, la salud, la disciplina, el desarrollo de rasgos de la personalidad y sus cualidades integrales, en el tratamiento a los problemas manifiestos y latentes en las relaciones grupales, entre otros.

Esas coordenadas armonizan con el funcionamiento de la interrelación de las configuraciones que integran esta dimensión Organizativa Práctico Laboral Investigativa, basa su esencia en el conjunto de precisiones, indicaciones y sugerencias profesionales desde una perspectiva formativa que contiene una intencionalidad confirmativa desde un enfoque educativo de preparación, cuya finalidad presupone, a partir de la formación psicopedagógica del estudiante, sean tomados en consideración aquellos problemas profesionales característicos de la atención a la diversidad educativa, contenido de obligatorio dominio para la dirección exitosa de las actividades en el marco de la Cultura Física y en particular en las clases de Educación Física.

Desde esta óptica, esta configuración descubre y resalta como necesidad esencial la orientación desde los contenidos de la Didáctica General y Particular, en las que se deben concebir diferentes formas de tratamiento al contenido atención a la diversidad educativa mediante la organización de la PLI, en función de los objetivos que se persiguen con su realización, basadas en la orientación precisa de los aspectos metodológicos que guiarán al estudiante en sus procederes preprofesionales. De ahí que se determina como segunda configuración Didáctico Práctico Laboral Investigativa, desde la que se insiste en hacer énfasis en la orientación de los componentes, recursos, herramientas didácticas que le permitirán saber operar con los conocimientos sobre atención a la diversidad educativa, comprender con claridad los aspectos epistemológicos y metodológicos básicos, elementales sobre atención a la diversidad educativa, esencialmente los recursos para su realización, motivarlos e idear el proceso de manera que pueda entender el antes, ahora y después; ello dará cuenta de un aspecto esencial para la organización de la PLI, el entendimiento pedagógico, categoría resultante que da cuenta del proceso subjetivo a través del cual se logra que los estudiantes 
tomen conciencia de la importancia, sentido y significado que tiene el ofrecer una atención a la diversidad educativa de calidad, lo que hace posible que el estudiante esté en mejores condiciones de aplicar adecuadamente los conocimientos asimilados; demostrando el desarrollo de habilidades pre profesionales, de sentimientos, actitudes y comportamientos coherentes con el respeto a lo heterogéneo, a lo diverso, en correspondencia con lo determinado en la segunda dimensión Aplicativa de la PLI.

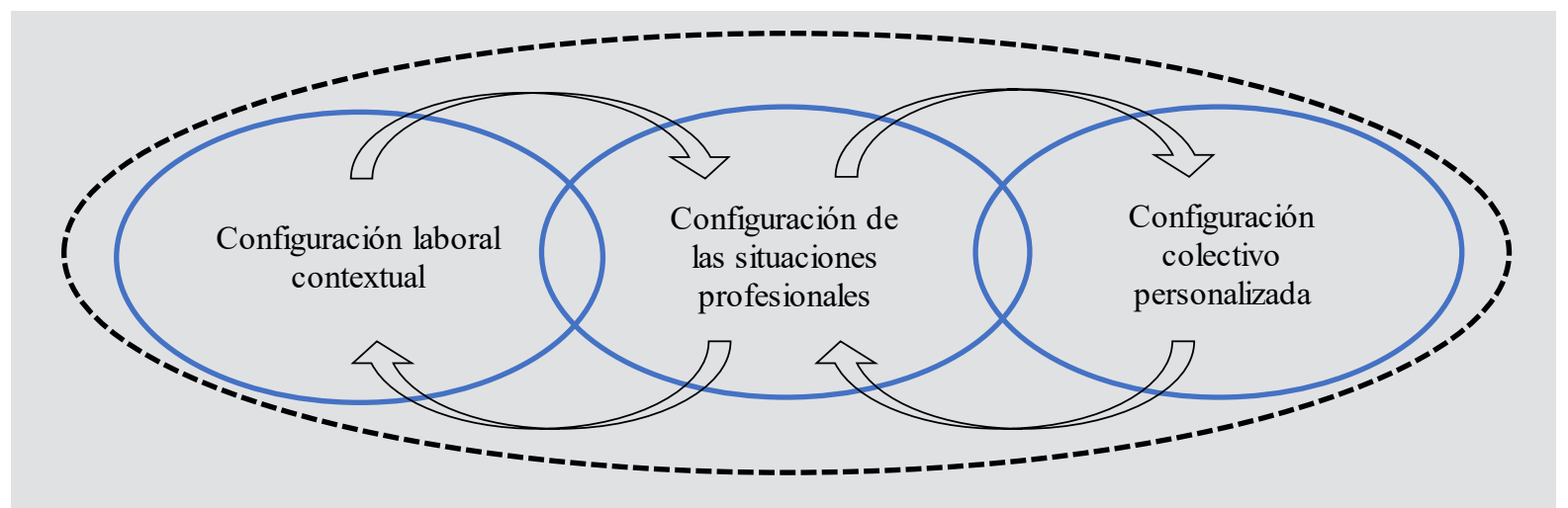

Figura 2. Esquema de la dimensión aplicativa de la PLI

La dimensión Aplicativa de la PLI responde a las perspectivas y expectativas del estudiante en el escenario laboral, el establecimiento de sus relaciones socio laborales, el intercambio con profesionales de experiencias, las vivencias, eventos, situaciones desde las que construye sus experiencias laborales, proceso que debe transcurrir bajo la guía orientadora del profesor encargado de esta actividad, el tutor u otros agentes implicados en el proceso.

Por lo tanto, estamos resignificando el valor de la entidad laboral base como contexto ideal para el crecimiento integral del estudiante, en el que se da la confrontación entre los conocimientos obtenidos y las realidades de la práctica frente a situaciones frecuentes y cambiantes, enriqueciendo más el proceso al intercambiar con los compañeros que actuaron en el mismo u otros escenarios laborales. Por eso, se determina la configuración Laboral Contextual donde se insiste en la importancia que tiene para el estudiante conocer en detalles las características, fortalezas y debilidades de los contextos de actuación, que va a hacer en ellos, por qué y para qué; sistematizando sus experiencias laborales, registrando los acontecimientos más significativos de las realidades de la práctica, sus vivencias, el sentido personal que le otorga y las correspondientes valoraciones.

Todo ello es expresión la significatividad contextual, o sea, del grado de significado que el estudiante le otorga al contexto en su formación inicial, enriqueciendo su crecimiento profesional y humano, el cual es posible cuando al organizar el proceso formativo se tiene en cuenta las particularidades de la entidad laboral base y su entorno comunitario, los recursos humanos, materiales y físico ambientales, de los cuales depende la manifestación de las diferentes situaciones profesionales que enfrenta y debe dar atención el estudiante. 
Se debe lograr la pertinencia entre las tareas de la práctica y del currículo, para posibilitar una relación espacio-tiempo sincrónica, que pueda articular los conocimientos sobre atención a la diversidad educativa con la práctica, el desempeño del estudiante y los procesos de confrontación entre conocimientos teóricos aprendidos y las experiencias vividas en la práctica preprofesional, la cual se desarrolla en un contexto social determinado, con características distintivas y problemas profesionales típicos de la dinámica de la vida de ese contexto profesional.

Todo este análisis permitió determinar una segunda configuración, denominada Contextualización de las Situaciones Profesionales, que alude al análisis y tratamiento de las situaciones profesionales de cada contexto educativo, donde se encuentran respuestas o soluciones pedagógicas basadas en la aplicabilidad valorativa, cualidad resultante que es expresión de la puesta en práctica de los conocimientos adquiridos en el componente académico sin divorciarse de las valoraciones de aquellas peculiaridades, potencialidades, barreras, entre otras, que caracterizan, identifican o tipifican ese escenario educativo particular. Por ello, se requiere que el estudiante rote por diferentes instituciones que garanticen una visión más completa del comportamiento de las situaciones educativas en diferentes contextos de actuación profesional para determinar con veracidad los problemas que inciden desde los escenarios de aprendizaje, los motivos y necesidades de los escolares desde su desarrollo físico-motor, afectivo, cognitivo, instrumental, ético, etc.

Determinándose así la tercera configuración denominada Colectivo Personalizada que establece en los espacios y acciones de la práctica tenga lugar la construcción colectiva e individualizada del conocimiento, mediado por la acción de profesionales de experiencia, el tutor y la incidencia del ambiente socio laboral, donde emergen experiencias y conocimientos esenciales, se formulan interrogantes referidas a qué debe y puede hacer, con qué recursos cuenta para ello, qué experiencias posee, entre otras. Todo ello constituye objeto de apropiación de conocimientos por el practicante, permitiéndole emitir, compartir y renovar los juicios de valor que ha ido conformando durante la PLI; cuestionamiento que da cuenta de la interactividad comunicativa cualidad resultante de la retroalimentación que se produce en el proceso comunicativo, demostrativo que se realiza en la PLI de Educación Física, donde el estudiante y el educando, los profesores y tutores, la familia, entre otros, intercambian información y se producen respuestas consientes entre ellos, para lograr mejores experiencias en lo que respecta a la atención a la diversidad educativa para el desarrollo integral del educando.

En ese permanente intercambio, donde se reafirma la adecuada orientación para el tratamiento al contenido atención a la diversidad educativa, se crean las condiciones objetivas y subjetivas en el proceso de la PLI para que florezcan interesantes y valiosas reflexiones, criterios; permitiendo al estudiante la reformulación de su actuación, de su desempeño; en tanto se va potenciando en él capacidades reflexivas para emitir valoraciones, juicios críticos, arribar a conclusiones sobre su desempeño en la práctica desde la atención a la diversidad educativa, aspectos que se determinan en la tercera dimensión Valorativa de la PLI. 


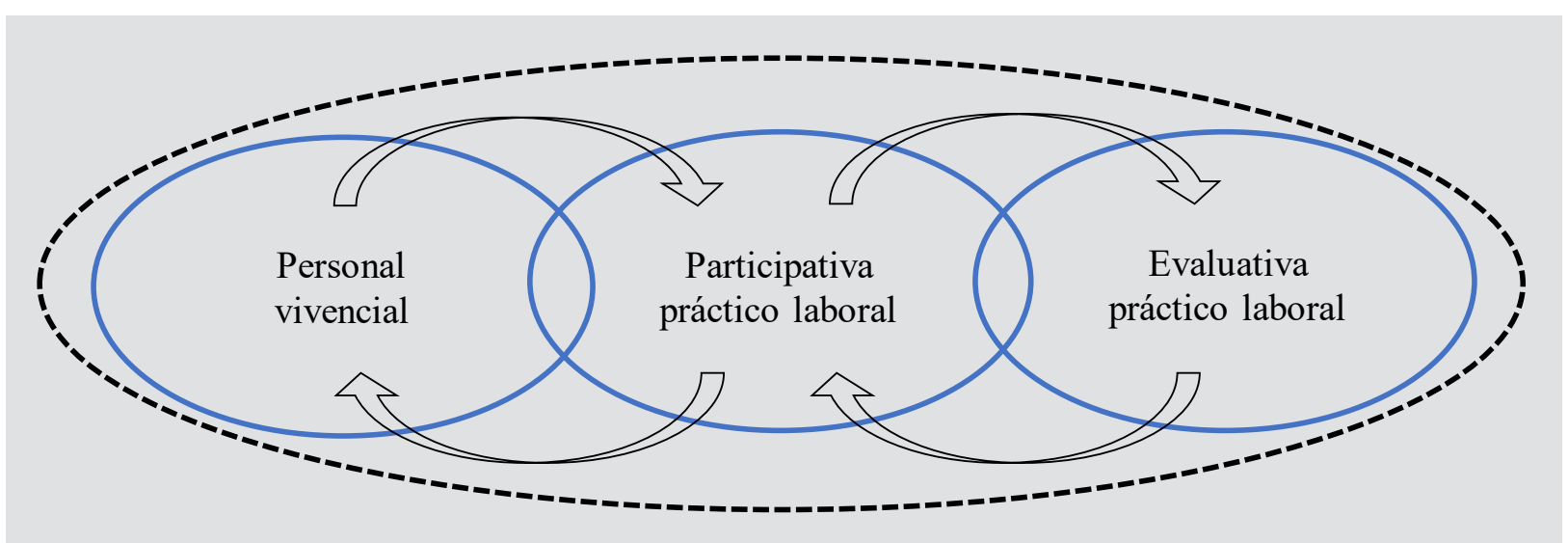

Figura 3. Esquema de la dimensión valorativa de la PLI

De aquí emerge la intencionalidad interpretativa de las regularidades del desarrollo de los educandos, cualidad que da cuenta de la actuación preprofesional del estudiante con un propósito hermenéutico que permita interpretar, comprender y explicar las particularidades del desarrollo de los escolares, de su entorno y del sistema de relaciones que establece en él; de manera que pueda ofrecer las respuestas pedagógicas necesarias y pertinentes en correspondencia con las necesidades educativas de estos y con los preceptos de la pedagogía de la diversidad y de la Educación Física, con una visión holística sobre la existencia de obstáculos en el proceso formativo, y la solución de los problemas educativos que ellos generan dentro de la diversidad educativa. Todo ese desempeño ético-valorativo va teniendo lugar desde la acumulación de experiencias individuales vivenciadas por el estudiante, el sentido que le concede, su significado, motivos, inquietudes y expectativas profesionales, acompañadas de las vivencias colectivas.

A partir de este análisis y asumiendo que lo vivencial es consustancial con la reconstrucción de conocimientos, experiencias, sentido significado, historias personales, espacios de criterios y valoraciones compartidas, se determinó como parte del proceso de preparación del estudiante para atender la diversidad educativa, una nueva configuración Personal Vivencial que resignifica el papel de las vivencias del individuo en la estructuración del desarrollo psicopedagógico del estudiante.

Esa asimilación y acumulación de vivencias personales del estudiante se produce durante su participación en las actividades de la práctica preprofesional, de ahí que se determina como otra configuración la Participativa Práctico Laboral que prescribe el aprovechamiento óptimo de los diferentes espacios donde se materializa la formación laboral-investigativa del estudiante. En tal sentido, se sugiere propiciar momentos, espacios y recursos que estimulen la participación de éste en cada una de las tareas integradoras planificadas, en las que sea capaz de dialogar, debatir y reflexionar sobre el alcance y dominio de sus modos de actuación pre profesional.

En estos espacios desempeña papel importante la interactividad comunicativa en la situación profesional, ya referida, la que vista desde esta configuración presupone la práctica de una comunicación con una marcada intencionalidad formativa de estilos de comunicación, y un mayor protagonismo del estudiante que le permita acceder al diálogo con las personas interactuantes, en las situaciones profesionales a las que debe contribuir a resolver. 
A medida que el estudiante alcanza mayor protagonismo en su quehacer y en la valoración de la calidad de la PLI realizada, se va adentrando en un proceso evaluativo de su crecimiento profesional y humano, por lo que va funcionando a tono con los presupuestos de la tercera configuración determinada, denominada Evaluación Práctico Laboral, que hace referencia a una evaluación como proceso de carácter regulador, individualizado, integral y flexible, que ponga énfasis en los aspectos que denotan un tratamiento a la diversidad educativa en el ámbito de la Cultura Física basada en la igualdad de oportunidades, la aceptación, el respeto y la tolerancia, para fomentar el desarrollo integral del educando. De esta manera, el estudiante se reconoce en un constante proceso de preparación para la toma de decisiones en la solución de los profesionales.

La evaluación implica valorar con facilidad la opinión, la voluntad o la actitud de los estudiantes, respetando las reglas, sin sujetarse a normas estrictas; es ser susceptible de cambios o variaciones según las circunstancias o necesidades, reconociendo que toda personalidad es única e irrepetible.

Se es del criterio, entonces, de que cuando el estudiante recibe, y es capaz de asimilar la base orientadora para su accionar en la PLI con el contenido atención a la diversidad educativa desde presupuestos de la Pedagogía de la Diversidad (Dimensión 1), asume modos de actuación coherentes con estos preceptos, aplicando principios pedagógicos como: el carácter colectivo e individual, el respeto a la personalidad del educando. Es capaz de expresar el grado de satisfacción que va experimentando a medida que aplica esos conocimientos (Dimensión 2), todo ello le permite ir evaluando, sistemáticamente sus modos de actuación profesional (Dimensión 3).

Los fundamentos de estas dimensiones del modelo son materializados en la metodología cuya implementación permite dar cuenta en la PLI, el dominio de una cultura pedagógica de atención a la diversidad educativa que lo pone en condiciones de mostrar un desempeño preprofesional diversificado, máxima expresión de la calidad del profesional de la cultura potencialmente transformador de modos y estilos de atención a la diversidad educativa en el marco de las clases de Educación Física.

\section{CONCLUSIONES}

La sistematización de los principales antecedentes investigativos e históricos revelan las inconsistencias, teóricas y metodológicas que orientan la investigación hacia la preparación de los estudiantes de tercer año de licenciatura en Cultura Física centrada en la atención a la diversidad educativa durante la Práctica Laboral Investigativa en Educación Física.

El modelo propuesto permite la preparación del estudiante que realiza su PLI en Educación Física con sede en la Secundaria Básica, lo que posibilita desde lo formativo y lo contextual la correspondencia con las configuraciones establecidas para resolver las contradicciones que generan el movimiento al interior del mismo; entonces emerge el desempeño preprofesional diversificado del estudiante de Cultura Física.

Los resultados avalan la instrumentación parcial de la metodología, así como su aprobación lo que permite sostener la propuesta como alternativa metodológica para preparar a los estudiantes en la atención a la diversidad educativa durante la realización de su Práctica Laboral Investigativa en Educación Física. 


\section{REFERENCIAS}

Addine, F. (1996). Alternativa para la organización de la práctica laboral investigativa en los ISP. (Doctorado). Instituto Superior Pedagógico Enrique José Varona.

Aldea, N. (2006). Los Resultados Científicos como aportes de la investigación educativa. Santa Clara: Instituto Superior Pedagógico Félix Varela. Centro de Ciencias e Investigaciones Pedagógicas, (59) 27-35.

Bermúdez, R. (2002). Teoría y metodología del aprendizaje. Pueblo y Educación.

Blanco, D. (2015). Concepción pedagógica del desarrollo de software educativo para la web. (Doctorado). Universidad de Ciencias Pedagógicas "José de la Luz y Caballero". https://repositorio.uho.edu.cu/jspui/bitstream/uho/5035/1/tes.pdf

Calzado, Z. (2004). Programa de Didáctica de la Matemática I. (Licenciatura) Universidad de Ciencias Pedagógicas Frank País García.

Castillejo, A. (2004). Estrategia de superación para promover el aprendizaje desarrollador en la Educación Física. Memorias Universidad 2008 [CD-ROM]. Pre-Congreso Pedagogía. La Habana. Universidad de La Habana.

Castillo, S. M. (2002). Currículum y formación de educadores. Conferencia Científico Metodológico. (pp. 11.23). Santiago de Cuba. Universidad de Ciencias Pedagógicas Frank País García.

Castillo, S. M.(2014). Modelo pedagógico de superación del profesional de la Cultura Física y el Deporte para la atención a la diversidad desde una perspectiva comunitaria. (Doctorado). Universidad de Ciencias Pedagógicas Frank País García.

Díaz, A. (2005). Las redes pedagógicas locales desde la perspectiva de la gestión del conocimiento. Formación continua de docentes. Un camino para compartir 20002005. Centro de Perfeccionamiento e Investigaciones Pedagógicas del Ministerio de Educación.

García, G. (2004). La formación laboral investigativa en los profesionales en formación. Pueblo y Educación.

García, G. (2005). Profesionalidad y práctica pedagógica. La Habana: Editorial Pueblo y Educación.

Guerra, D. (2005). Teoría de la motivación y práctica profesional. Editorial Pueblo y Educación.

Hechavarría, M. (2006). La educación Física y los valores para la atención a la diversidad deportiva. (Doctorado). Editorial Comisión Nacional de Deportes en México.

Jaur. L. (2017). Educar a los protagonistas del futuro: Revista Correo de la UNESCO.

Marchesi, A. (2015). Calidad de la enseñanza en los tiempos de cambio. Editorial Alianza.

Mataran, A. C. (2015). Concepción didáctica para el protagonismo del estudiante en el proceso de formación inicial del Licenciado en Cultura Física. (Doctorado). Universidad de Oriente. 
Mendoza, E. (2007). Modelo Didáctico de la Formación Interdisciplinaria del Licenciado en Cultura Física. (Doctorado). Universidad de Oriente.

Milán, S. (2005). Las habilidades profesionales como proceso pedagógico permanente en el entrenador deportivo. Congreso Internacional Pedagogía 2001. (pp. 29-33). La Habana: Universidad de La Habana.

Moreno, M.J. (2003). Una concepción pedagógica de la estimulación motivacional en el proceso de enseñanza aprendizaje. (Doctoral). Instituto Superior Pedagógico "Enrique José Varona".

Novoa, M. (2006). Formación continua de Educadores desde sus contenidos en torno a la función cultural de la familia: Nuevos Desafios. Organización de Estados Iberoamericanos para la Educación, la Ciencia y la Cultura.

Oroceno, M. (2008). La formación didáctica del entrenador deportivo para la atención a la diversidad. (Doctoral). Universidad de Oriente.

Prada, D. (2014). Evolución del concepto de atención a la diversidad. (Licenciatura). Universidad de Valladolid. https:/uvadoc.uva.es/bitstream/handle/10324/8404/ TFG-O\%20399.pdf? sequence=1\&isAllowed $=\mathrm{y}$

Rabilero, H. R. (2010). La motivación profesional pedagógica en los estudiantes de la Licenciatura en Cultura Física: una metodología para su orientación. (Doctorado). Universidad de Ciencias Pedagógicas Frank País García.

Ramírez, G. (2009). Estrategia Pedagógica para la implementación de la Cultura Física en condiciones de universalización de los institutos superiores pedagógicos. (Doctorado). Centro de Estudios Pedagógicos “Juan Bautista Sagarra Blez".

Robert, R.E. (2009). Estrategia educativa para potenciar la educación de la autovaloración en futuros profesionales de la Cultura Física. (Doctorado). Instituto Superior de Cultura Física "Manuel Fajardo".

Suárez, C. (2003). Enfoque integral de la formación del profesional de cara a los desafíos del S XXI. En [CD-ROM]. Evento Provincial Pedagogía 2005. Santiago de Cuba. Dirección Provincial de Educación.

Vaillant, A. (2001). Algunos modelos importantes en la investigación pedagógica. ICCPMES.

Velázquez, E. (2003). La práctica laboral en el proceso de universalización de los Institutos Superiores Pedagógicos. Pedagogía 2003. La Habana. Universidad de La Habana.

Venet, R. (2007). La evaluación de impacto en la Investigación Educativa. Reflexiones. Universidad de Ciencias Pedagógicas Frank País García.

Vinent, M. (2000). Estrategia educativa para el desarrollo de la autodeterminación en el proceso de formación integral de los estudiantes preuniversitarios. (Doctoral). Universidad de Ciencias Pedagógicas Frank País García. 